\title{
Polycystic ovary syndrome and increased risk of psychiatric disorders
}

\begin{abstract}
Aim: This study is aimed to assess, for the first time the prevalence of polycystic ovary syndrome (PCOS) and associated psychological disorders among women at reproductive age in Mississippi.

Methods: The data was collected through "the Patient Cohort Explorer" system at the University of Mississippi Medical Center (UMMC) from January 2013 to December 2018. De-identified patients were searched with diagnosis code for PCOS, age, ethnicity, and associated diagnosis including weight gain, anxiety, depression, attention-deficit/ hyperactivity disorder (ADHD), and other psychological concerns.

Results: Collected data from 166,748 females (19-45 years) showed 1.4\% (95\% CI: 1.32 - 1.44) prevalence of PCOS in women seen in UMMC clinics during the period of study. Almost $51 \%$ of patients with PCOS suffered from one or more psychological disorders; anxiety $21 \%$ (95\% CI: 19.3 - 22.6), depression $20 \%$ (95\% CI: $18.9-22.1)$, ADHD 3.2\% (95\% CI: $2.6-4.0)$, and bipolar disorder $2.5 \%$ (95\% CI: $1.9-3.2)$. In addition, prevalence values suggest a positive correlation between obesity, anxiety, and depression in PCOS patients.

Conclusion: Results from this study provide 1) for the first time an estimate regarding the prevalence of PCOS and associated psychological disorders in women with PCOS in Mississippi, 2) associated psychological disorders in PCOS women may be diverse based on race and ethnicity. Our data clearly highlight that the psychological well-being of women with PCOS are affected. Therefore, it is critical for the primary care and specialty clinics to use appropriate psychological screenings. Left undiagnosed and/or untreated, chronic psychological disorders may exacerbate physical and mental health conditions.
\end{abstract}

Keywords: anxiety, attention-deficit/hyperactivity disorder, bipolar disorder, depression, mississippi, polycystic ovary syndrome
Volume 8 Issue 6 - 2020

\author{
Mohadetheh Moulana PhD, ${ }^{1,2}$ Anju P \\ Sukumaran $\mathrm{MD}^{3}$ \\ 'Departments of Psychiatry and Human Behavior, USA \\ ${ }^{2}$ Women's Health Research Center, USA \\ ${ }^{3}$ Pediatric-Endocrinology, University of Mississippi Medical \\ Center, USA
}

\author{
Correspondence: Mohadetheh Moulana, PhD, Division of \\ Neurobiology and Behavior Research, Department of Psychiatry \\ and Human Behavior, University of Mississippi Medical Center, \\ 2500 North State Street, Jackson, MS 39216, USA, \\ Tel 60I-984-6696, Fax 60I-984-693I, \\ Emailmmoulana@umc.edu
}

Received: December 16, 2020 | Published: December 29, 2020

\section{Introduction}

Polycystic ovary syndrome (PCOS), is a common endocrine malfunction affecting $9-18 \%$ of women of reproductive age, based on different diagnostic criteria used. ${ }^{1-3}$ PCOS is characterized by hyperandrogenism, chronic oligo/anovulation, and polycystic ovaries. PCOS is associated with a variety of comorbidities such as metabolic disorders, obesity, insulin resistance, and chronic inflammation. ${ }^{4-6}$ Moreover, growing compelling data indicate that women with PCOS suffer from anxiety, depression, social phobia, suicidal ideation, bipolar disorder, and attention-deficit/hyperactivity disorder (ADHD).$^{7-15}$ The prevalence of anxiety and depression in women with PCOS is greater compared to age-matched non-PCOS women $(29 \%-50 \%$ vs $18 \%)$ and $\left(57 \%\right.$ vs $7 \%$ ), respectively ${ }^{7,9,10,16}$ and the body of evidence indicates that the prevalence of depression in PCOS women is greater than that reported for women with other chronic diseases including coronary artery disease (18\%), lupus (26\%), and diabetes (28\%). ${ }^{17-19}$ In addition, women with PCOS are at higher risk of being overweight or obese,${ }^{20}$ and obesity increases not only the risk of metabolic disorders and cardiovascular diseases $\mathrm{s}^{6,21}$ but also it is considered as a hallmark of long-term depression risk in PCOS populations. ${ }^{22,23}$

It has been shown that the longer it takes to receive a diagnosis of PCOS, the more likely women are to be depressed or anxious ${ }^{7,10,16}$ and the presence of an anxiety disorder increases risk of later major depressive disorder ${ }^{24}$ The chronic nature and high incidence of anxiety and depression is a substantial public health burden. Therefore early detection and treatment of mental adverse effects in PCOS patients is crucial.

In spite of the compelling data that indicate the prevalence of psychological comorbidities in women with PCOS in different geographical areas and the position statement from "the Androgen Excess and PCOS Society" in 2018 which highlighted the necessity of further research, ${ }^{25}$ only one study with a sample size of 10 women is found in Mississippi. ${ }^{26}$ Not only is the prevalence of psychological disorders in PCOS women lacking, but also there is no data to show the prevalence of PCOS in Mississippi, which experiences significant health disparities.

The aim of the current study was to address these gaps in the existing literature on PCOS occurrence and associated psychological disorders among diverse populations in Mississippi by investigating: first the prevalence of PCOS in a group of women that have been seen in UMMC clinics, second the occurrence of associated psychological disorders, specifically anxiety, depression, and ADHD among the group, and third whether these psychological disorders are more common in a subgroup of overweight/obese PCOS patients. To the best of our knowledge, this is the first study that evaluates the prevalence of PCOS and the impact of PCOS on women mental health in Mississippi. 


\section{Methods}

De-identified data were collected through "the Patient Cohort Explorer" system at the University of Mississippi Medical Center. The application is a research tool through the electronic health record, EPIC, which supports rapid querying and reporting that facilitates obtaining a list of unidentified patients based on either patient encounter, or data filters. The de-identified data was collected from women with PCOS $(n=2300)$ who were seen in clinics at UMMC from January 2013 to December 2018.

PCOS was diagnosed by the presence of two of the three following criteria: hyperandrogenism, ovulatory dysfunction, and polycystic ovaries. The diagnosis code for PCOS (ICD-10 E28.2) was used to search the unidentified patients, followed by demographic factors like age, ethnicity, and race. Then extra diagnosis codes for abnormal weight gain, anxiety, depression, bipolar disorder, ADHD, and behavioral concerns were added to the filtered data. Inclusion criteria included diagnosis of PCOS based on medical records and being at reproductive age; $19-45$ years old. The primary analysis investigated the differences between reproductive age women with and without PCOS.

\section{Statistical analysis}

The prevalence estimates and $95 \%$ confidence intervals were calculated using the propCI function from the $\mathrm{R}$ statistical package "prevalence". ${ }^{27}$ All calculations were done in $\mathrm{R}$ version 3.5.3. ${ }^{28}$

\section{Results}

Data showed that from 166,748 females (19-45) years old that were seen at UMMC clinics during 6 years of study. A total of 2,300 had a diagnosis of PCOS, 1,032 were African Americans, 1,104 White, and $7 \%$ other minorities (Table 1). The prevalence rate of PCOS among the population was 1.4\% (95\% CI: 1.32-1.44), African Americans $1.19 \%$ (95\% CI: $1.12-1.27)$, and White 1.9\% (95\% CI: $1.81-2.03)$.
Data indicated that $45 \%$ (95\% CI: 43-47) of the PCOS patient seen at UMMC were African Americans and 49\% (95\% CI: 43 - 56) were White.

Table I De-identified data from the "Patient Cohort Explorer" system

\begin{tabular}{llll}
\hline & $\begin{array}{l}\text { Total } \\
\text { population }\end{array}$ & $\begin{array}{l}\text { African } \\
\text { American }\end{array}$ & White \\
\hline Female 19-45years & 166,748 & 86472 & 57606 \\
Anxiety & 14657 & 5894 & 7983 \\
Depression & 13871 & 6316 & 6888 \\
Weight Gain + Anxiety & 596 & 256 & 321 \\
Weight Gain + Depression & 574 & 284 & 276 \\
PCOS & 2300 & 1032 & 1104 \\
PCOS + Anxiety & 480 & 147 & 307 \\
PCOS + Depression & 470 & 169 & 278 \\
PCOS + Weight Gain & 207 & 90 & 102 \\
PCOS + Weight Gain + Anxiety & 57 & 15 & 40 \\
PCOS + Weight Gain + & 54 & 17 & 36 \\
Depression & & & \\
\hline
\end{tabular}

Data showed that the anxiety and depression were significantly higher in age-matched PCOS patients compared with women without PCOS; anxiety [21\% (95\% CI: $19.3-22.6)$ vs $8.8 \%$ (95\% CI: $8.7-$ $8.9)$ ], depression [20\% (95\% CI: $18.9-22.1)$ vs $8 \%$ (95\% CI: $8.2-$ $8.5)]$. Anxiety and depression were observed to be more common in White at [64\% (95\% CI: 59.6-68.1)] and [59\% (95\% CI: 54.6-63.5)], respectively in contrast to [31\% (95\% CI: $26.7-34.9)]$ and $36 \%(95 \%$ CI: $31.7-40.4)$ ] in African Americans (Table 2).

Table 2 Prevalence of PCOS and Psychological Disorders among women 19-45 years old in Mississippi (Females, $n=166,748 ;$ PCOS, $n=2300$ ). Abbreviation; Weight Gain (Wt)

\begin{tabular}{|c|c|c|c|}
\hline \multirow{3}{*}{ Female (19-45) } & Total Population & African American & White \\
\hline & prevalence rate & prevalence rate & prevalence rate \\
\hline & $(95 \% \mathrm{Cl})$ & $(95 \% \mathrm{Cl})$ & $(95 \% \mathrm{Cl})$ \\
\hline PCOS & $1.4(1.32-1.44)$ & $1.2(1.12-1.27)$ & $1.9(I .8 I-2.03)$ \\
\hline PCOS + Anxiety & $21(19.3-22.6)$ & 31 (26.7-34.9) & $64(59.6-68.1)$ \\
\hline PCOS + Depression & $20(\mid 8.9-22.1)$ & $36(31.7-40.4)$ & $59(54.6-63.5)$ \\
\hline $\mathrm{PCOS}+\mathrm{Wt}$ & $9(7.7-10.2)$ & $43(36.9-50.3)$ & $49(42.5-56.0)$ \\
\hline PCOS $+W t+$ Anxiety & $28(21.9-34.0)$ & $26(16.7-39)$ & $70(57.3-80.5)$ \\
\hline PCOS + Wt + Depression & $26(20.6-32.5)$ & $3 I(20.7-44.7)$ & $67(53.4-77.8)$ \\
\hline Non-PCOS + Wt & $1.30(1.2-1.4)$ & $57(55.0-59.2)$ & $38.5(36.5-40.6)$ \\
\hline Non-PCOS + Anxiety & $8.8(8.7-8.9)$ & $40(39.4-41.0)$ & $54(53.0-54.7)$ \\
\hline Non-PCOS + Depression & $8.3(8.2-8.5)$ & $46(44.7-46.4)$ & $50(48.8-50.5)$ \\
\hline Non-PCOS + Wt + Anxiety & $0.36(0.33-0.39)$ & $43(39.0-46.0)$ & $54(49.8-57.8)$ \\
\hline Non-PCOS + Wt + Depression & $0.34(0.32-0.37)$ & $49(45.4-53.6)$ & $48(44.0-52.2)$ \\
\hline
\end{tabular}


Furthermore, data indicated that $9 \%$ of the patients with PCOS $(n=207)$ were overweight. Fifty seven $[28 \%(95 \%$ CI; $21.9-34)]$ of the overweight subset were diagnosed with anxiety and 54 [26\% (95\% CI: 20.6-32.5)] with depression. Moreover, anxiety and depression were more common in overweight White patients with $[70 \%$ (95\% CI: $57.3-80.5)$ ] and [67\% (95\% CI: 53.4-77.8)], respectively compared to [26\% (95\% CI: 16.7-39)] and [31\% (95\% CI: 20.7-44.7)] in African American overweight PCOS women, respectively (Table 2).

In addition, it was found that $10 \%$ of patients were diagnosed with other psychological concerns such as ADHD [3.2\% (95\% CI: 2.6-4.0)], bipolar disorder [2.5\% (95\% CI: 1.9-3.2)] suicidal ideation [1.3\% (95\% CI: $0.9-1.9)]$, and other behavioral disorders [2.7\% (95\% CI: $2.0-3.4)]$.

\section{Discussion}

The present study, is the first to show the prevalence rate of PCOS and the associated psychological comorbidities in a group of women with PCOS in Mississippi. Results of our study indicated that the prevalence rate for PCOS in the women of reproductive age (19-45 years) seen at UMMC clinics is lower not only compared to Spain $(6.5 \%),{ }^{29} \operatorname{Iran}(6.8 \%),{ }^{30}$ and Southern China $(2.2 \%),{ }^{31}$ but also compared to its neighbor in the South in the United States; Alabama $(4.6 \%))^{32}$ Unfortunately, limited assessment of PCOS has been documented at different geographical regions in the United States. While, Okoroh and colleagues have reported significantly higher PCOS prevalence rate in Southern US (47\%), North Central (23.0\%), West $(18.7 \%)$, and the North East $(10.3 \%),{ }^{33}$ the lower estimate in Mississippi may be due to variety of factors, such as heterogeneity features of PCOS and having high percentage of undiagnosed women with PCOS in the community due to the existing health disparities in Mississippi and lack of specialist access.

PCOS is heterogeneous syndrome with multifactorial pathophysiology, and its prevalence varies among different ethnicities and across various geographical and social communities ranging from $2.2 \%$ to $26 \%$ worldwide. ${ }^{34}$ The prevalence rate as low as $2.4 \%$ in Sothern China or as high as $21 \%$ in Australian indigenous women may suggest the impact of race and ethnicity on the prevalence and features of PCOS. ${ }^{3,35-38}$ However, the influence of race and ethnicity remains controversial since Wolf and colleagues in a review of the existing studies across the globe concluded that the prevalence of PCOS is not influenced by race or ethnicity. In the current study we found that there were slight differences, which were not significant between racial groups with respect to the prevalence of PCOS, African American and White patients seen at UMMC clinics with a prevalence of $1.2 \%$ and $1.9 \%$, respectively. The result is consistent with the previous studies. ${ }^{31,32}$

Remarkably, our results indicated a significant increase of anxiety and depression prevalence rate in women with PCOS in Mississippi compared to the age-matched non-PCOS women. Our results support the association of mood disorders with PCOS as reported in previous studies. ${ }^{7,8,10,16,39-41}$ In the present study the association between anxiety, depression and PCOS was found to be varied by race and ethnicity. Anxiety and depression were significantly higher in White patients compared to African Americans with PCOS which may suggest that the psychological impact of PCOS is diverse based on race and ethnicity, which support the concept of heterogeneous expression of the same psychopathological diseases in PCOS, particularly when our data indicate that anxiety and depression in non-PCOS African American women is $40 \%$ (39.4-41.0) and $46 \%$ (44.7-46.4), respectively which are significantly higher compared to PCOS African Americans.
Whether lower percentage of African American PCOS patients with anxiety and depressive symptoms, is a biological phenomenon similar to the metabolic phenotype of PCOS that has been reported by Engmann and colleagues that African American women with PCOS exhibit milder phenotype, ${ }^{42}$ is unknown and requires further examination. Furthermore, the lower percentage may not suggest that PCOS have less impact on the development of psychological disorders in African American women than White, and it could be due to variety of factors, such as the difference in the diagnosis of psychological disorders, stigma of being diagnosed with psychological disorders that prevent them to disclose symptoms, and/or due to the increased barriers to access and receiving mental health care for patients who are racial/ethnically diverse. More prospective studies are needed to further examine this finding. Noteworthy that our study was inclusive of all women seen in UMMC clinics and would be less likely to be impacted by socioeconomic and health status.

Our results showed that only $9 \%$ of the women with PCOS were overweight. Noteworthy, the prevalence of obesity in PCOS patients is highly fluctuating and is different according to race, ethnicity, geographic regions, and diagnostic criteria ranging from $6 \%$ to $100 \%{ }^{4,20,22}$ However, there is no comprehensive study to demonstrate overall prevalence of obesity in PCOS patients. In the current study the prevalence values suggest an association between weight and psychological disorders in overweight women at the reproductive age with PCOS in Mississippi which is consistent with previous reports. ${ }^{10,22}$ Despite the association of obesity with depression ${ }^{43}$ the diagnosed psychological disorders in women with PCOS are not solely the result of obesity, several factors may be contributing to the development and progression of the above-mentioned disorders. As our findings showed significant percentage of normal-weight PCOS women suffer from anxiety and depression, supporting the previous report which showed that women with PCOS are at higher risk for depression regardless of BMI. ${ }^{44}$

Finally, our results indicated that $3.2 \%$ of women with PCOS had diagnosis of ADHD which support the previous study that women with PCOS have higher ADHD symptoms. ${ }^{45}$ Moreover, this is consistent with many studies that suggest a role for androgen in the etiology of ADHD such as demonstration of dopamine innervation reduction in the frontal cortex and increased risk of ADHD-like behaviors in rats by exposure to excess androgen levels in early development. ${ }^{46}$ More studies in human and animal models are required to establish the role of androgen in the etiology of ADHD and PCOS.

\section{Limitation}

The findings in this study are subject to some limitations. The major limitation is the lack of access to the patients directly for more clinical examination; instead collecting the data via the de-identified medical record only. In addition, data is limited to the patients that have been diagnosed with PCOS in UMMC clinics only which limits the generalizability of the findings to the entire population in Mississippi and United States. Third, African American women may be reluctant to discuss and report mental health symptoms and pursue therapy due to the associated shame and stigma with such issues. Fourth, there may be high percentage of undiagnosed women with PCOS in the community due to the existing health disparities in Mississippi and lack of specialist access. These limitations suggest that the results should be considered as preliminary data and further research is required to establish causality and to understand the underlying risk factors of associated psychological disorders in PCOS in order to plan appropriate interventions to reduce psychological comorbidities in women with PCOS in Mississippi. 


\section{Implications for practice and/or policy}

Despite limitations, the findings of our study provide important directions for practice and/or policy. Taking into consideration that our results highlighted the anxiety and depression as two major psychological disorders that affected PCOS women, an appropriate psychological screening by primary care and specialty medical providers, such as endocrinologist and Obstetrics \& Gynecology clinics are strongly recommended. In addition to screening for psychological symptoms, the results of our study raise awareness that there is a need to develop targeted intervention strategies to decrease the prevalence of anxiety and depressive symptoms in women with PCOS in Mississippi.

\section{Conclusion}

In conclusion, from the current study, it is evident that due to the high prevalence of anxiety and depression in women with PCOS at reproductive age, the physical, emotional condition, and psychological concerns of these patients should be discussed and addressed in numerous ways to prevent further complications and manifestations. Health care providers in primary care, endocrinology, and Obstetrics \& Gynecology clinics must consider mental and emotional assessment and screening of women with PCOS in addition to the regular physical and biochemical examination, in order to evaluate and identify potential psychological disorders. Thereafter, identified patients must be referred to specialists for further evaluation, diagnosis, counseling sessions, wellness programs, and treatment. This study will help researchers and clinicians to work toward addressing psychological and emotional well-being of this vulnerable group of patients.

\section{Acknowledgments}

None.

\section{Ethical approval}

The project does not meet the definition of human subject research. In the current study only existing, de-identified, un-linkable data was reviewed. Therefore, IRB approval was not obtained.

\section{Informed consent}

In the current study only existing, de-identified, un-linkable data was reviewed. Therefore, informed consent was not required.

\section{Funding}

This research did not receive any specific grant from funding agencies in the public, commercial, or not-for-profit sectors.

\section{Conflicts of interest}

No conflicts of interest, financial or otherwise, are declared by the authors.

\section{References}

1. Diamanti-Kandarakis E, Kouli CR, Bergiele AT, et al. A survey of the polycystic ovary syndrome in the Greek island of Lesbos: hormonal and metabolic profile. Journal of Clinical Endocrinology and Metabolism. 1999;84:4006-4011.

2. Azziz R, Woods KS, Reyna R, et al. The prevalence and features of the polycystic ovary syndrome in an unselected population. Journal of Clinical Endocrinology and Metabolism. 2004;89:2745-2749.
3. Kauffman RP, Baker VM, Dimarino P, et al. Polycystic ovarian syndrome and insulin resistance in white and Mexican American women: A comparison of two distinct populations. American Journal of Obstetrics and Gynecology. 2002;187(5):1362-1369.

4. Conway GS, Agrawal R, Betteridge DJ, Jacobs HS. Risk factors for coronary artery disease in lean and obese women with polycystic ovary syndrome. Obstetrical \& Gynecological Survey. 1993;48(2):132-134.

5. Azziz R, Carmina E, Dewailly D, et al. Position statement: Criteria for defining polycystic ovary syndrome: An Androgen Excess Society guideline. Journal of Clinical Endocrinology and Metabolism. 2006;91:4237-4245.

6. Orio F, Vuolo L, Palomba S, et al. Metabolic and cardiovascular consequences of polycystic ovary syndrome. Minerva Ginecol. 2008;60(1):39-51.

7. Deeks A, Gibson-Helm, Paul E, et al. Is having polycystic ovary syndrome (PCOS) a predictor of poor psychological function including anxiety and depression? Human Reproduction. 2011;26(6):1399-1407.

8. Benson S, Hahn S, Tan S, et al. (2009). Prevalence and implications of anxiety in polycystic ovary syndrome: Results of an internet-based survey in Germany. Human Reproduction. 2009;24(6):1446-1451.

9. Sheps D, Sheffield D. Depression, anxiety, and the cardiovascular system: a cardiologist's perspective. Journal of Clinical Psychiatry. 2001;62(8):216.

10. Hollinrake E, Abreu A, Maifeld M, et al. Increased risk of depressive disorders in women with polycystic ovary syndrome. Fertility and Sterility. 2007;87(6):1369-1376.

11. Dokras A, Clifton S, Futterweit W, et al. Increased prevalence of anxiety symptoms in women with polycystic ovary syndrome: Systematic review and meta-analysis. Fertility and Sterility. 2012;97(1).

12. Annagür BB, Tazegül A, Uguz F, et al. Biological correlates of major depression and generalized anxiety disorder in women with polycystic ovary syndrome. Journal of Psychosomatic Research. 2013;74(3):244247.

13. Scaruffi E, Gambineri A, Cattaneo S, et al. Personality and psychiatric disorders in women affected by polycystic ovary syndrome. Frontiers in Endocrinology. 2014.

14. Klipstein KG, Goldberg JF. Screening for bipolar disorder in women with polycystic ovary syndrome: A pilot study. Journal of Affective Disorders. 2006;91(2-3):205-209.

15. Hergüner S, Harmanc1 H, Toy H. Attention deficit-hyperactivity disorder symptoms in women with polycystic ovary syndrome. The International Journal of Psychiatry in Medicine. 2015;50(3):317-325.

16. Mansson M, Holte J, Landin-Wilhelmsen K, et al. Women with polycystic ovary syndrome are often depressed or anxious-a case control study. Psychoneuroendocrinology. 2008;33:1132-1138.

17. Connerney I, Shapiro PA, Mclaughlin JS, et al. Relation between depression after coronary artery bypass surgery and 12-month outcome: A prospective study. The Lancet. 2001;358(9295):1766-1771.

18. Jump RL, Robinson ME, Armstrong AE, et al. Fatigue in systemic lupus erythematosus: contributions of disease activity, pain, depression, and perceived social support. Journal of Rheumatology. 2005;32(9):16991705.

19. Anderson RJ, Freedland KE, Clouse RE, et al. The prevalence of comorbid depression in adults with diabetes: a meta-analysis. Diabetes Care. 2001;24(6):1069-1078.

20. Lim S, Davies M, Norman R, et al. Overweight, obesity and central obesity in women with polycystic ovary syndrome: A systematic review and meta-analysis. Human Reproduction Update. 2012;18(6):618-637. 
21. Teede H, Deeks A, Moran L. Polycystic ovary syndrome: A complex condition with psychological, reproductive and metabolic manifestations that impacts on health across the lifespan. BMC Med. 2010;8(1):41.

22. Moran L, Gibson-Helm M, Teede H, et al. Polycystic ovary syndrome: A biopsychosocial understanding in young women to improve knowledge and treatment options. Journal of Psychosomatic Obstetrics \& Gynecology. 2010;31(1):24-31.

23. Greenwood EA, Pasch LA, Shinkai K, et al. Clinical course of depression symptoms and predictors of enduring depression risk in women with polycystic ovary syndrome: Results of a longitudinal study. Fertility and Sterility. 2019;111(1):147-156.

24. Parker G, Hadzi-Pavlovic D. Is the female preponderance in major depression secondary to a gender difference in specific anxiety disorders? Psychological Medicine. 2004;34(3):461-470.

25. Dokras A, Stener-Victorin E, Yildiz BO, et al. Androgen excess-polycystic ovary syndrome society: Position statement on depression, anxiety, quality of life, and eating disorders in polycystic ovary syndrome. Fertility and Sterility. 2018;109(5):888-899.

26. Bruce Laurel A. Body mass and depression among mississippi women with polycystic ovarian syndrome. Hattiesburg, MS: University of Southern Mississippi; 2014.

27. Devleesschauwer B, Torgerson P, Charlier J, et al. Prevalence: Tools for prevalence assessment studies. R package version. 2014.

28. R Core Team. A language and environment for statistical computing. Vienna, Austria: R Foundation for Statistical Computing; 2019.

29. Asunción M, Calvo RM, San Millán JL, et al. A prospective study of the prevalence of the polycystic ovary syndrome in unselected Caucasian women from Spain. Journal of Clinical Endocrinology and Metabolism. 2000;85:2434-2438.

30. Jalilian A, Kiani F, Sayehmiri F, et al. Prevalence of polycystic ovary syndrome and its associated complications in Iranian women: A metaanalysis. Iran Journal of Reproduction Medicine. 2015;13(10):591-604.

31. Chen X, Yang D, Mo Y, et al. Prevalence of polycystic ovary syndrome in unselected women from southern China. Eur J Obstet Gynecol Reprod Biol. 2008;139:59-64.

32. Knochenhauer ES, Key TJ, Kahsar-Miller M, et al. Prevalence of the polycystic ovary syndrome in unselected black and white women of the Southeastern United States: A prospective study. Journal of Clinical Endocrinology and Metabolism. 1998;83(9):3078-3082.

33. Okoroh EM, Hooper WC, Atrash HK, et al. Prevalence of polycystic ovary syndrome among the privately insured, United States, 2003-2008. American Journal of Obstetrics and Gynecology. 2012;207(4).
34. Yildiz BO, Bozdag G, Yapici Z, et al. Prevalence, phenotype and cardiometabolic risk of polycystic ovary syndrome under different diagnostic criteria. Human Reproduction. 2012;27(10):3067-3073.

35. Goodarzi MO, Quiñones MJ, Azziz R, et al. Polycystic ovary syndrome in Mexican-Americans: Prevalence and association with the severity of insulin resistance. Fertility and Sterility. 2005;84(3):766-769.

36. Al-Fozan H, Al-Futaisi A, Morris D, et al. Insulin responses to the oral glucose tolerance test in women of different ethnicity with polycystic ovary syndrome. Journal of Obstetrics and Gynaecology Canada. 2005;27(1):33-37.

37. Welt CK, Arason G, Gudmundsson JA, et al. Defining constant versus variable phenotypic features of women with polycystic ovary syndrome using different ethnic groups and populations. Obstetrical \& Gynecological Survey. 2007;62(3):185-187.

38. Wolf W, Wattick R, Kinkade O, et al. Geographical prevalence of polycystic ovary syndrome as determined by region and race/ethnicity. International Journal of Environmental Research and Public Health. 2018;15(11):2589.

39. Weiner CL, Primeau M, Ehrmann DA. Androgens and mood dysfunction in women: comparison of women with polycystic ovarian syndrome to healthy controls. Psychosomatic Medicine. 2004;66(3):356-362.

40. Adali E, Yildizhan R, Kurdoglu M, et al. The Relationship between clinicobiochemical characteristics and psychiatric distress in young women with polycystic ovary syndrome. Journal of International Medical Research. 2008;36(6):1188-1196.

41. Cesta CE, Månsson M, Palm C, et al. Polycystic ovary syndrome and psychiatric disorders: Co-morbidity and heritability in a nationwide Swedish cohort. Psychoneuroendocrinology. 2016;73:196-203.

42. Engmann L, Jin S, Sun F, et al. Racial and ethnic differences in the polycystic ovary syndrome metabolic phenotype. American Journal of Obstetrics Gynecology. 2017;216(5):493.e1-493.e13.

43. Luppino FS. Wit LM, Bouvy PF, et al. Overweight, obesity, and depression a systematic review and meta-analysis of longitudinal studies. Archives General Psychiatry. 2010;67(3):220-229.

44. Dokras A, Clifton S, Futterweit W, et al. Increased risk for abnormal depression scores in women with polycystic ovary syndrome. Obstetrics \& Gynecology. 2011;117(1):145-152.

45. Hergüner S, Harmanc1 H, Toy H. Attention deficit-hyperactivity disorder symptoms in women with polycystic ovary syndrome. The International Journal of Psychiatry in Medicine. 2015;50(3):317-325.

46. King JA, Barkley RA, Delville Y, et al. Early androgen treatment decreases cognitive function and catecholamine innervation in an animal model of ADHD. Behavioral Brain Research. 2000;107(1-2):35-43. 